Benchmarks

\title{
A simple method to generate stable cell lines for the analysis of transient protein-protein interactions
}

Emilia Elizabeth Savage, Denise Wootten, Arthur Christopoulos, Patrick Michael Sexton and Sebastian George Barton Furness

Drug Discovery Biology, Monash Institute of Pharmaceutical Sciences, Monash University, Parkville, Victoria, Australia

BioTechniques 54:217-221 (April 2013) doi 10.2144/000114013

Keywords: BRET; flpIN; Gateway; protein interaction; bicistronic vector; arrestin; GPCR

Transient protein-protein interactions form the basis of signal transduction pathways in addition to many other biological processes. One tool for studying these interactions is bioluminescence resonance energy transfer (BRET). This technique has been widely applied to study signaling pathways, in particular those initiated by $\mathrm{G}$ protein-coupled receptors (GPCRs). These assays are routinely performed using transient transfection, a technique that has limitations in terms of assay cost and variability, overexpression of interacting proteins, vector uptake limited to cycling cells, and non-homogenous expression across cells within the assay. To address these issues, we developed bicistronic vectors for use with Life Technology's Gateway and flpIN systems. These vectors provide a means to generate isogenic cell lines for comparison of interacting proteins. They have the advantage of stable, single copy, isogenic, homogeneous expression with low inter-assay variation. We demonstrate their utility by assessing ligand-induced interactions between GPCRs and arrestin proteins.

Bioluminescence Resonance Energy Transfer (BRET) is a popular method for monitoring transient protein-protein interactions in live cells. It has been widely applied to study interactions between $G$ protein-coupled receptors (GPCRs) and their interacting proteins such as $G$ proteins, arrestins, G proteincoupled receptor kinases (GRKs) and other GPCRs $(1,2)$.
This assay relies on the fusion of genetically encoded Renilla luciferase (RLuc) donor and green fluorescent protein (GFP) acceptor proteins to the interacting partners. To monitor interactions, cDNA chimeras encoding interacting partners (fused with donor and acceptor) are routinely prepared in separate plasmids and transiently co-transfected prior to the assay.
Transient transfection assays can exhibit wide inter-assay variation due to variable transfection efficiency and may be costly in high-throughput formats, depending on the transfection reagent used. Transient transfections also typically result in very high transgene expression, potentially leading to a high baseline BRET signal or a low signal-to-noise ratio in ligandinduced BRET due to a high level of non-specific (collisional) interactions. In addition, overexpression can significantly alter the pharmacological behavior of receptors. During transient transfection, only a subpopulation of cells are transfected and there is a significant cell-cycle bias for DNA uptake, which has the potential to skew results of interaction studies (3).

To overcome the limitations of transient transfection and establish a reliable method for isogenic expression of interacting proteins, we designed bicistronic BRET vectors that take advantage of Life Technologies' (Carlsbad, California, USA) Gateway cloning and flpIN cell line systems. These vectors are based on the pEF5/ FRT/V5-DEST flpIN destination vector from Life Technologies. This vector yields stable incorporation into a single (isogenic) site in the genome of flpIN cell lines. The EFI $\alpha$ promoter that drives expression of the bicistronic transcript is mammalian, rather than viral in origin, and provides stable expression. The encephalomyocarditis virus (EMCV) internal ribosome entry site (IRES) was chosen as this has been demonstrated to harbour true IRES activity in mammalian cells (4). It was placed upstream of the acceptor $\mathrm{cDNA}$ fusion as it has been shown to drive 7 to 10 -fold greater expression of the second cistron (5). Thus the acceptor fusion will be in excess compared to the donor, minimizing the bystander BRET effect (6). We call these vectors BIVISTI for $\underline{B}$ RET IRES vector for isogenic stable incorporation to monitor transient interaction.

The parental pEF5/FRT/V5-DEST vector contains a V5 epitope tag downstream of the second recombination site and is flanked by BstBI and $P m e I$ restriction sites. We designed an

\section{Method summary:}

Here we present a series of bicistronic vectors based on the Gateway and flpIN systems which enable the rapid generation of isogenic cell lines for protein-protein interaction assays. As proof of principle, we assess ligand-induced interactions between $G$ protein-coupled receptors and arrestin proteins generated via isogenic cell lines. 

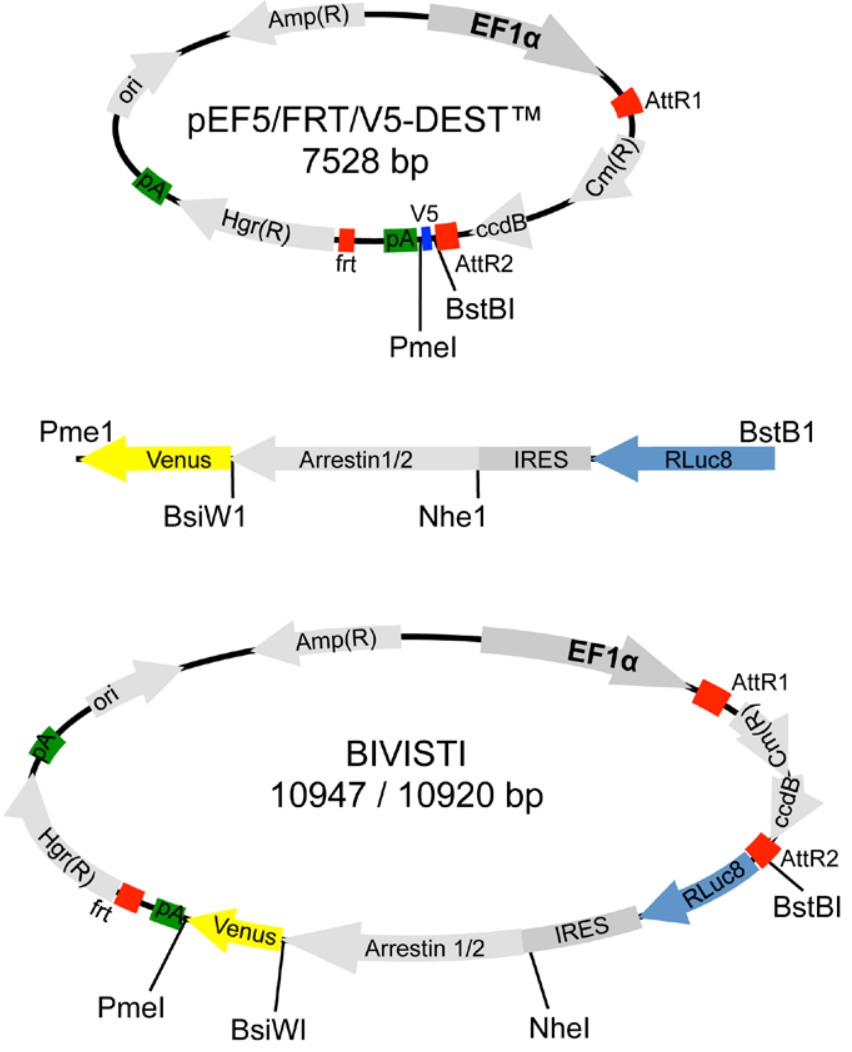

Figure 1. Scheme for replacement of the V5 epitope tag of parental vector pEF5/FRT/V5-DEST with the bicistronic BRET insert to generate a BIVISTI vector. 5' BstBI and 3' Pmel sites corresponding to those flanking the V5 epitope flank the bicistronic insert. The bicistronic insert comprises the Renilla luciferase variant RLuc8 followed by the ECMV IRES sequence, $\beta$-arrestin 1 or 2 fused to the GFP variant Venus. Key vector features are indicated using standard contractions.

insert flanked by Bst BI and PmeI sites that would replace this sequence with one containing a cassette with the coding sequence for $R L u c 8$ (GenBank: EF446136.1) followed by the unattenuated EMCV IRES sequence (nucleotides 149-713 relative to the polyprotein start site of Gen Bank: DQ288856) and a coding sequence for either $A R R B 1$ ( $\beta$-arrestin 1, GenBank: NM_004041.3) or ARRB2 ( $\beta$-arrestin 2, GenBank: NM_004313.3) in frame with the GFP variant Venus (GenBank: DQ092360). The RLuc8 sequence was placed in reading frame $B$, relative to the Gateway cassette, yielding a 26 amino acid linker when used with a stop codondeleted coding sequence from a Gateway entry vector. The linker sequence is: DPAFLYKVVDIQHSGGRSSLEGPRFE and is predicted to form a mixture of extended and coil secondary structure (7). The native start codon from the EMCV IRES was retained, followed by an NheI site to allow conventional cloning of the acceptor fusion partner then the start- and stop codon-deleted coding sequence of $A R R B I$ or 2 followed by a $B s i \mathrm{WI}$ site and start codon-deleted coding sequence of Venus (Figure 1).

To validate these vectors, stop codon-deleted sequences for 3 distantly related GPCRs, GLP1R (glucagon-like peptide 1 receptor), CHRM1 (muscarinic 1, acetylcholine receptor), and AVPR2 (vasopressin 2 receptor) were cloned into pENTR 11 and subsequently into the BIVISTI vectors by Gateway cloning, with GLP1R having an N-terminal cMyc epitope tag immediately following the signal peptide (8). Stable flpIN CHO cell lines were established by standard methods using these vectors. We then assayed these receptors for $\mathrm{G}$ protein-dependent function in calcium mobilisation or cAMP accumulation assays, confirming pharmacology consistent with untagged receptors in this cell background (an example of this is shown in Figure 2E). We then assessed the ability of these constructs to report transient recruitment of arrestin proteins to these receptors by ligand-induced BRET as described previously (9). We performed 3-4 independent time course experiments on stably transfected cells of various passage numbers ranging from 17 to 35 . In addition, parallel transient transfection using the AVPR2/ARRB2 BIVISTI construct was performed. BRET readings were collected using a LUMIstar Omega instrument (BMG Labtech Ortenberg, Germany) that allows sequential integration of signals detected in the 465-505 and 515-555 $\mathrm{nm}$ windows using filters with the appropriate band pass. The BRET signal was calculated by subtracting the ratio of $515-555 \mathrm{~nm}$ emission over 465-505 $\mathrm{nm}$ emission for a vehicle-treated cell sample from the same ratio for the ligand-treated cell sample (ligandinduced BRET). This background-subtracted mean data from these 3-4 experiments are shown in Figure 2. In response to stimulation by $1 \mu \mathrm{M}$ arginine vasopressin of the AVPR2/ ARRB2 cell line we saw a mean ligand-induced increase of 87 \pm 3.2 milliBRET units (Figure 2A). This was slightly larger than the ligand-induced increase observed in parallel transient transfection of AVPR2/ARRB2 (71 \pm 4 milliBRET), which also showed more point to point variability. Stimulation of the CHRM1/ARRB2 cell line with $100 \mu \mathrm{M}$ acetylcholine produced a mean ligand-induced increase of $16.2 \pm 3.3$ milliBRET (Figure 2A and B). In response to stimulation of the GLP1R/ARRB2 cell line with 100nM GLP-1(7-36)NH2 we saw a mean ligand-induced increase of $24 \pm 2.1$ milliBRET (Figure 2A and C). The ligand-induced change in milliBRET units for GLP1R is relatively small in comparison with the strongly coupled AVPR2; however, the response is highly consistent with small errors, allowing for the construction of a concentration response curve for the GLP1R/ARRB2 cell line in response to GLP-1(7-36)NH2 (Figure 2D). This concentration response curve was generated from the peak ligand-induced BRET values from 4 independent experiments and was fitted to a sigmoid dose-response curve using PRISM (GraphPad Software, La Jolla, California, USA) to yield a $\mathrm{pEC}_{50}$ for ARRB2 recruitment of $7.5 \pm 0.1$ with an $\mathrm{R}^{2}$ of 0.92 (Figure 2D). To examine our ability to detect differences in arrestin recruitment patterns, we generated CHRM1/ARRB1 and GLP1R/ARRB1 stably transfected cell lines. In contrast to the CHRM1/ARRB2 cell line, there was no acetylcholinedependent recruitment of ARRB1 to CHRM1, although GLP-1(7-36)NH2-dependent recruitment of ARRB1 to GLP1R was observed with a maximum ligand-induced BRET increase of $24 \pm 3$ milliBRET units (Figure $2 F$ ). To examine the correlation between receptor and $\beta$-arrestin expression, the GLP1R/ARRB2 was subjected to flow cytometry. Briefly, cells were harvested in versene (PBS + 0.5mM EDTA) and stained using AF647 (Life Technologies)-conjugated 9E10 (monoclonal against the cMyc epitope, produced in-house by standard methods, with degree of labeling $=4.6)$ at $2 \mathrm{ug}$ / $\mu \mathrm{L}$ and Sytox blue (Life Technologies) for live/dead discrimination. Data were collected on a FACSCantoII (BD Biosciences, San Jose, California, USA) and analyzed using FlowJo (Tree Star, Ashland, Oregon, USA). As a control, flpIN CHO cells expressing untagged GLP1R were stained and analyzed 
A

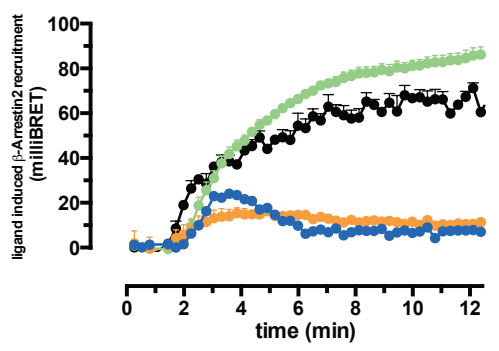

D

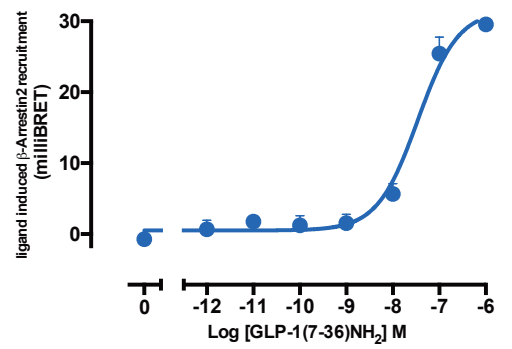

B

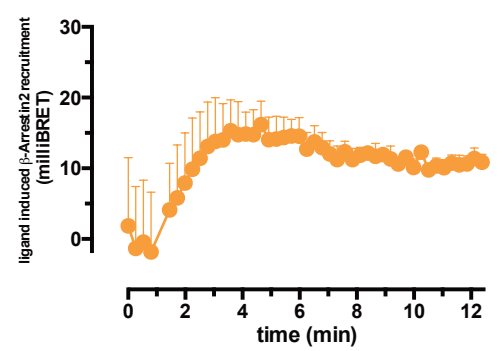

E

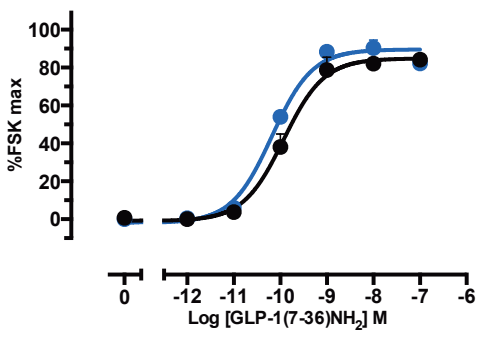

C

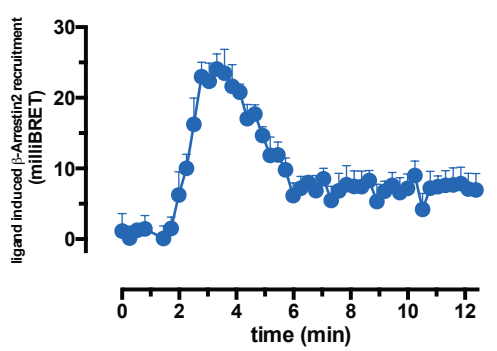

$\mathbf{F}$

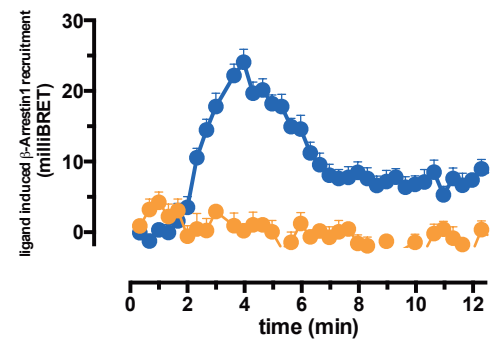

Figure 2. Ligand-induced recruitment of ARRB2 \& ARRB1 to AVPR2, GLP1R and CHRM1 receptors. (A), Stable flpIN CHO cell lines expressing AVPR2-RLuc8/ ARRB2-Venus (green), CHRM1-RLuc8/ARRB2-Venus (orange), GLP1R-Rluc8/ARRB2-Venus (blue) or flpIN CHO cells transiently transfected with AVPR2/ ARRB2-Venus (black) and stimulated with $1 \mu \mathrm{M}$ arginine vasopressin for AVPR2-RLuc8/ARRB2-Venus, 100 $\mu \mathrm{M}$ acetylcholine for CHRM1-RLuc8/ARRB2Venus or 100nM GLP-1(7-36)NH2 for GLP1R-Rluc8/ARRB2-Venus. Data shown are mean \pm SEM from 3-4 independent experiments performed in triplicate over passages 17 to 35. Peak ligand induced milliBRET responses for ARRB2 recruitment are $87 \pm 3.2$ (AVPR2), $16.2 \pm 3.3$ (CHRM1), $24 \pm 2.1$ (GLP1R), and $71 \pm 4$ (AVPR2 transient), respectively. (B) and (C) are the same as A but with expanded y-axis shown for CHRM1-RLuc8/ARRB2-Venus (B) and GLP1RRluc8/ARRB2-Venus (C). (D), concentration response curve for GLP-1(7-36)NH2 induced recruitment of ARRB2 in the stable flpIN CHO, GLP1R-Rluc8/ ARRB2-Venus cell line. Data are fit to the three-parameter logistic equation. The $\mathrm{R}^{2}$ for the curve fit is 0.92 with a calculated $\mathrm{pEC} \mathrm{C}_{50}$ of $7.5 \pm 0.1$. Data are the mean \pm SEM of four independent experiments conducted in triplicate. (E), comparison of concentration response curve for GLP-1(7-36)NH2 stimulated cAMP accumulation in GLP1R (black) and GLP1R-Rluc8/ARRB2 (blue) flpIN CHO stable cell lines. Data are normalized to the maximum cellular response to forskolin (\%FSK max) and fit to the three-parameter logistic equation. The $\mathrm{pEC}_{50}$ values are $9.9 \pm 0.1$ and $10.2 \pm 0.1$. Data are the mean \pm SEM of four independent experiments conducted in triplicate. (F), stable flpIN CHO cell lines expressing CHRM1-RLuc8/ARRB1-Venus (orange) or GLP1R-Rluc8/ARRB1Venus (blue) were respectively stimulated with $100 \mu \mathrm{M}$ acetylcholine or 100nM GLP-1(7-36)NH2. Data shown are mean \pm SEM from 3-4 independent experiments performed in triplicate over passages 17 to 35. No ligand induced ARRB1 recruitment is evident for CHRM1, whereas GLP1R showed a peak recruitment of are $24 \pm 3$ milliBRET in response to 100nM GLP-1(7-26)NH2.

A

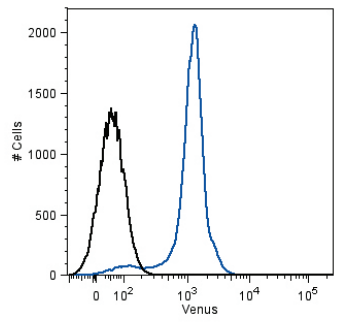

D

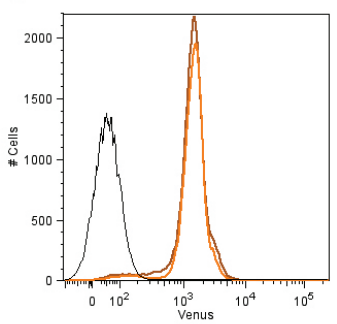

B

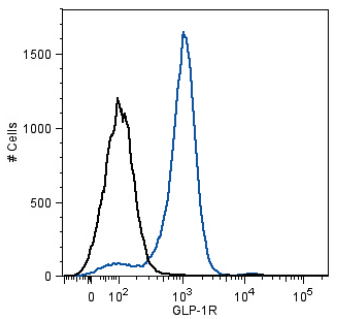

E

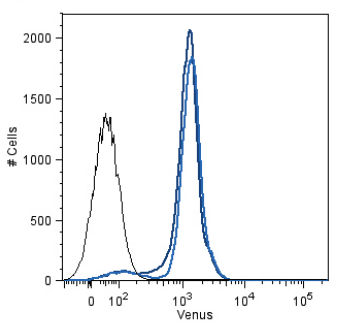

C

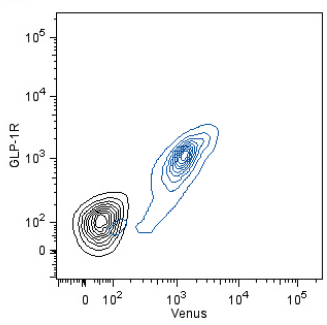

$\mathbf{F}$

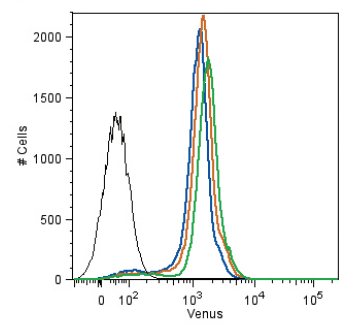

Figure 3. Flow cytometric analysis of GLP1R and ARRB2-Venus expression in stable fIpIN CHO cells. Untagged GLP1R (black) and CMycGLP1R-Rluc8/ARRB1-Venus (blue) flpIN CHO cells were stained with AF647-9E10 and Sytox blue and live cells analyzed for Venus and cMycGLP$1 R$ expression [(A), (B) and (C)]. (A) and (B) are histograms of relative fluorescence intensity of Venus and AF647-9E10, respectively, with the density plot in (C) showing relative fluorescence intensity of Venus plotted against that of AF647-9E10. (D) is a histogram of the relative fluorescence intensity of Venus from the CHRM1/ ARRB2 flpIN $\mathrm{CHO}$ cell line at passage 17 (light orange) and 35 (dark orange) with stained, untagged GLP1R flpIN CHO as the control (black). $(E)$ is a histogram of the relative fluorescence intensity of Venus from the GLP1R/ARRB2 fIpIN $\mathrm{CHO}$ cell line at passage 17 (light blue) and 35 (dark blue) with stained, untagged GLP1R fIpIN $\mathrm{CHO}$ as the control (black). (F) is a histogram of the relative fluorescence intensity of Venus from the AVPR2/ARRB2 (green), CHRM1/ARRB2 (orange) and GLP1R/ARRB2 (blue) fIpIN $\mathrm{CHO}$ cell lines with stained, untagged GLP1R fIpIN CHO as the control (black). 
in parallel. Figure 3 shows the distribution of expression as a histogram plot for direct fluorescence from the Venustagged ARRB2 (Figure 3A, blue) and cMyc-tagged GLP1R-RLuc8 (Figure 3B, blue). The direct correlation of ARRB2 and GLP1R expression is demonstrated in the contour plot in Figure 3C (blue). The stability of expression over time was assessed by analysis of Venus fluorescence of the CHRM1/ARRB2 and GLP1R/ARRB2 cell lines at passage 17 and 35 (Figure 3D, orange and E, blue). A comparison of ARRB2-Venus expression between AVPR2/ARRB2 (green), CHRM1/ARRB2 (orange), and GLP1R/ARRB2 (blue) cell lines was also performed with stained untagged GLP1R cells as a control (black)(Figure 3F). Consistent with previous reports including Reference 10, stably transfected flpIN CHO cell lines show a narrow, single mode distribution of transgene expression. These plots are representative of three independent experiments.

In conclusion, we report a simplified scheme for generation of stable cell lines expressing both donor and acceptor fusion partners for protein-protein interaction studies by BRET. The incorporation of Gateway technology for the donor fusion makes this a useful tool for the development of cell lines for screening GPCRs and GPCR small molecule libraries for interactions with arrestin and other partner proteins. In addition, we have confirmed that this system also works with the distantly related Class C GPCR calcium sensing receptor (CaSR). The NheI and BsiWI sites flanking the acceptor protein allow facile replacement of these fusions. We have developed BIVISTI variants containing GRK acceptor fusions and have been able to demonstrate robust recruitment of four isoforms of these kinases to GLP1R. The fact that cell lines generated using this system are isogenic also makes it a useful tool to examine structure-activity relationships of receptor mutants for partner protein interactions. This is a simple and robust system that should be amenable to a wide variety of applications and will assist in improving assay-to-assay variation as well as the general shortcomings associated with transient transfection.

\section{Acknowledgements}

PMS and AC are Principal Research Fellows of the National Health and Medical Research Council of Australia
(NHMRC). This work was funded by the NHMRC program grant \#519461

\section{Competing interests}

The authors declare no competing interests.

\section{References}

1. Kocan, M. and K.D.G. Pfleger. (2011) Study of GPCR-protein interactions by BRET. Methods Mol Biol. 746:357-371.

2. Jaeger, W.C., K.D.G. Pfleger, and K.A. Eidne. (2010) Monitoring GPCR-Protein Complexes Using Bioluminescence Resonance Energy Transfer. In D.R. Poyner, M. Wheatley(Eds.), G Protein-Coupled Receptors: Essential Methods. WileyBlackwell, Chichester, West Sussex, UK.

3. Rodriguez, A. and E.K. Flemington. 1999. Transfection-mediated cell-cycle signaling: considerations for transient transfectionbased cell-cycle studies. Anal. Biochem. 272:171-181.

4. Bert, A.G., R. Grépin, M.A. Vadas, and G.J. Goodall. 2006. Assessing IRES activity in the HIF-1alpha and other cellular 5D UTRs. RNA 12:1074-1083.

5. Bochkov, Y.A. and A. Palmenberg. 2006. Translational efficiency of EMCV IRES in bicistronic vectors is dependent upon IRES sequence and gene location. BioTechniques. 41:283-284.

6. Mercier, J.F., A. Salahpour, S. Angers, A. Breit, and M. Bouvier. 2002. Quantitative assessment of $\beta 1$-and $\beta 2$-adrenergic receptor homo-and heterodimerization by bioluminescence resonance energy transfer. J. Biol. Chem. 277:44925-44931.

7. Petersen, B., T.N. Petersen, P. Andersen, M. Nielsen, and C. Lundegaard. 2009. A generic method for assignment of reliability scores applied to solvent accessibility predictions. BMC Struct. Biol. 9:51.

8. Koole, C., D. Wootten, J. Simms, C. Valant, R. Sridhar, O.L. Woodman, L.J. Miller, R.J. Summers, et al. 2010. Allosteric ligands of the glucagon-like peptide 1 receptor (GLP-1R) differentially modulate endogenous and exogenous peptide responses in a pathway-selective manner: implications for drug screening. Mol. Pharmacol. 78:456-465.

9. Willard, F.S., D. Wootten, A.D. Showalter, E.E. Savage, J. Ficorilli, T.B. Farb, K. Bokvist, J. Alsina-Fernandez, et al. 2012. Small molecule allosteric modulation of the glucagon-like Peptide-1 receptor enhances the insulinotropic effect of oxyntomodulin. Mol. Pharmacol. 82:1066-1073.

10. Karimi, M., L.C. Goldie, D. Ulgiati, and L.J. Abraham. 2007. Integration site-specific transcriptional reporter gene analysis using Flp recombinase targeted cell lines. BioTechniques. 42:217-224.

Received: 16 October 2012; accepted: 27 February 2013

Address correspondence to Sebastian George Barton Furness, Drug Discovery Biology, Monash Institute of Pharmaceutical Sciences,
Monash University, Parkville, Victoria, Australia. E-mail: sebastian.furness@monash.edu

To purchase reprints of this article, contact: biotechniques@fosterprinting.com 